\title{
Non-invasive anaerobic threshold measurement using fuzzy model interpolation
}

\author{
John V. Ringwood ${ }^{1}$, James O’Neill ${ }^{1}$, Peter Tallon ${ }^{2}$, Neil Fleming ${ }^{3}$ and Bernard Donne ${ }^{4}$
}

\begin{abstract}
The interface between skeletal muscle activation through aerobic and anaerobic glycolysis is of key interest to sportspeople and athletes who participate in medium to long distance sports, such as middle- and long-distance running, cycling, swimming, rowing, kayaking and a variety of other events. To date, the gold standard for measuring anaerobic threshold (AT) is a structured test to exhaustion where blood lactate concentration is measured at regular intervals. However, the need for invasive testing, requiring trained personnel and specialist equipment, limits the availability of such tests. This paper proposes a non-invasive AT measurement method, which validates well against AT measured using lactate analysis. In addition, the proposed test has a relatively loose set of requirements on the exercise test protocol required and just requires a measure of exercise intensity and heart-rate. While the test is applicable to a range of sports, usage is demonstrated in this paper for a set of cyclists, using velocity as a measure of exercise intensity.
\end{abstract}

\section{INTRODUCTION}

Anaerobic threshold (AT) refers to the point at which the predominant energy supply system in the body changes from aerobic (with oxygen) to anaerobic (without oxygen). We use the term 'predominant' here to indicate that the transition from aerobic to anaerobic is not immediate, but happens gradually. Fig.1 shows the amount of energy supplied by the different energy systems with progressive increase in performance time. In Region 1, corresponding to short duration activities such as 100-200m sprinting, Andenosine Triphosphate (ATP), the basic chemical which effects muscular contraction, is supplied via stored ATP with additional ATP supplied using anaerobic degradation of glycogen (anaerobic glycolysis), which produces lactic acid as a by-product. In Region 2, for example involving 400m running, ATP is predominantly supplied via the lactic acid system. Region 3 sees a predominant mix of lactic acid and aerobic systems, while activities in Region 4 (e.g. marathon running) almost exclusively use the aerobic system, where ATP is supplied via the aerobic conversion of glycogen (aerobic glycolysis) [1], [2].

Clearly, athletes need to train specific energy system in order to optimise athletic performance and, in particular,

\footnotetext{
${ }^{1}$ John Ringwood and James O'Neill are with the Dept. of Electronic Eng., National University of Ireland Maynooth, Co. Kildare, Ireland (e-mail: john.ringwood@eeng.nuim.ie,jamesoneill12@yahoo.com)

${ }^{2}$ Peter Tallon is with Workday, Fumbally Square, Fumbally Lane, Dublin 8, Ireland (email: peter.tallon@workday.com)

${ }^{3}$ Neil Fleming is with the Dept. of Kinesiology, Recreation and Sport, Indiana State University Terre Haute, Indiana, USA (email: neil.fleming@indstate.edu)

${ }^{4}$ Bernard Donne is with the Dept. of Physiology, Trinity College Dublin, Ireland (e-mail: b.donne@mail.tcd.ie)
}

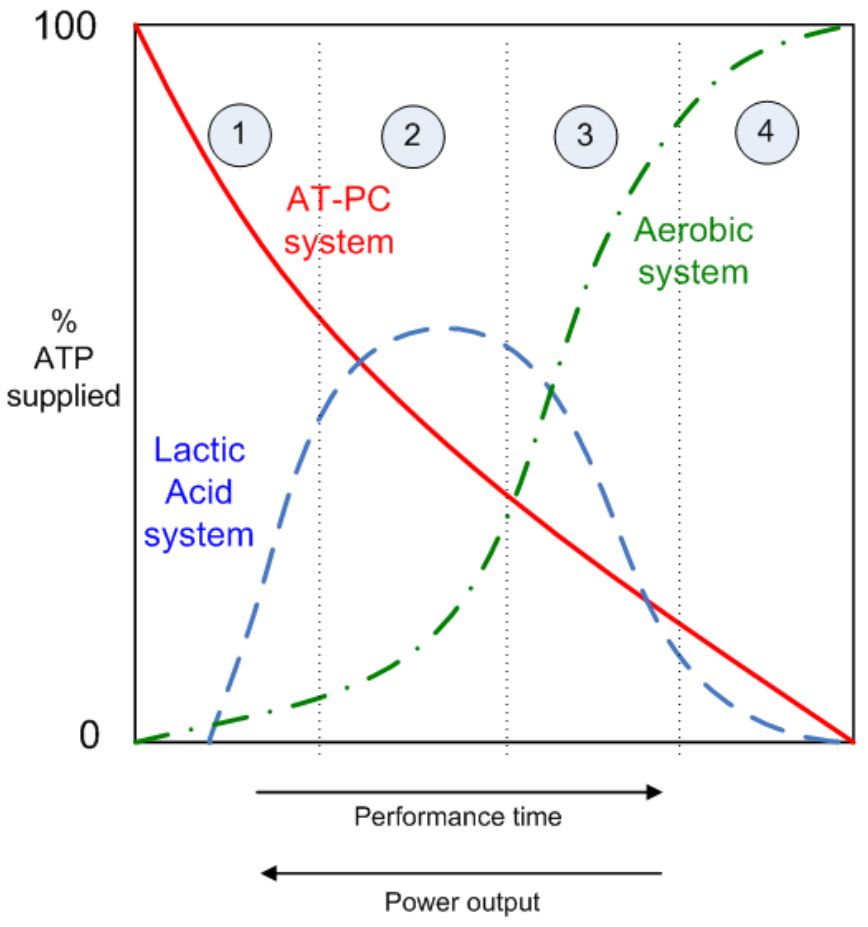

Fig. 1. Energy supply systems for exercise

the boundary between aerobic and anaerobic production of ATP is of significance. For athletes whose focus is aerobic events, the production of lactic acid, which happens relatively quickly, is highly undesirable since it impairs performance and is slow to disperse. Thus, knowledge of this boundary, or anaerobic threshold (AT) is key, if optimum training and performance is to be achieved. The AT is usually specified either in terms of a specific exercise intensity e.g. running/cycling speed, or the steady-state heart rate associated with such an exercise intensity. With modern sports instrumentation [3], it is relatively straightforward to have a real time measure of both exercise intensity and heart-rate.

Various scientific measures for AT have been proposed. The somewhat ideal Maximal Lactate Steady State (MLSS) [4], [5] identifies the exercise intensity point at which the rate of lactic acid production starts to exceed that of dispersion, resulting in a progressive increase in lactic acid. However, the difficulty of an athlete retaining a constant exercise rate to establish steady state lactic acid conditions, for a large number of exercise intensity points, makes such a physical test relatively impractical, resulting in a number of alternatives [5]. A closely related measure to MLSS is Onset of Blood Lactate Accumulation (OBLA) [6], which typically 
adopts a threshold (e.g. $4 \mathrm{mmol} / \mathrm{L}$ of lactate) to identify AT. Ultimately, the current gold standard in AT measurement involves the performance of a structured exercise programme under controlled conditions in an attempt to establish quasi steady-state conditions at a number of exercise intensities and physical measurement of lactate, a salt closely connected with production of lactic acid, concentration in the blood, is sampled at each nominal intensity. Such experimental conditions demand specialised equipment (exercise ergometer, lactate analyser), a strict exercise protocol (which may be difficult to achieve) and specialist medical personnel to take blood samples.

Some non-invasive methods have been determined for AT determination, the best known of which is Conconi's method [7], [8], which is not without its sceptics [9], [10] and also suffers from an inherent difficulty in achieving steady-state conditions [11]. However, the concept that the relationship between exercise intensity and heart-rate is roughly proportional up to the point of AT and nonlinear beyond AT is interesting and provides the basic hypothesis for the current work.

In this paper, we identify linear and nonlinear dynamical models for the relationship between exercise intensity and heart-rate, corresponding to regions of lower and higher exercise intensity, respectively. A Takagi-Sugeno-Kung (TSK) [12] fuzzy interpolation between models is performed, with that crossover point which corresponds to the overall model minimum mean squared modelling error (MSE) returning the non-invasive AT measure. The results from this method are validated against AT measurements from lactate analysis.

The remainder of the paper is organised as follows: Section II shows the modelling methodology, while Section III show the test protocols followed in recording the experimental data and how the data were processed. The model results, validated against experimental AT determination, are shown in Section IV, while conclusions are drawn in Section V.

\section{FUZZY MODEL INTERPOLATION}

The overall concept of fuzzy interpolation of models is articulated in Fig.2. Linear and nonlinear models, representing the relationship between exercise intensity and heart rate, are determined for data corresponding to low and high exercise intensities, respectively. Fuzzy interpolation is carried out between the models and optimal values for $p_{1}$ anD $p_{2}$, in an overall minimum model mean square error (MSE) are determined. The point at which the transition occurs from linear to nonlinear, i.e. $\left(p_{1}+p_{2}\right) / 2$, is taken as the nominal anaerobic threshold. In reality, steady state data is rarely achieved. In the case of the original Conconi test, where runners are required to run around a $400 \mathrm{~m}$ track, timing their arrival with 2 'beepers' placed at 200 intervals which set the pace, there is inevitably some constant speed adjustment from the athlete in trying to arrive at the exact instants of the audible 'beeps'. If a treadmill is used, a steady pace is not also exactly achieved, due to the constant adjustment of a speed control loop for the treadmill belt, in

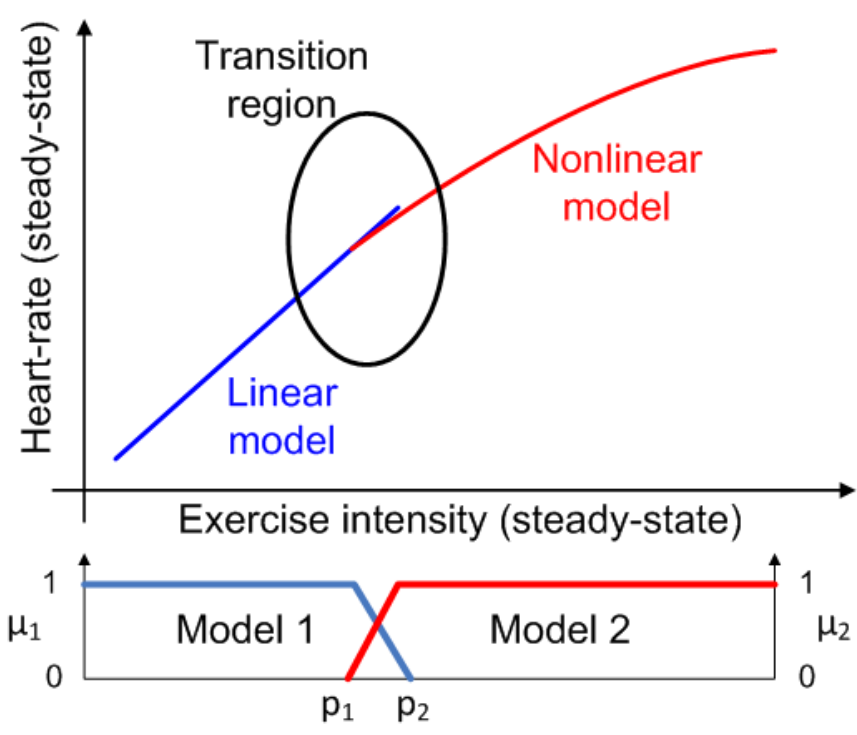

Fig. 2. Fuzzy interpolation concept

response to footfalls and other disturbances [11]. In the use of cycling/kayak/rowing ergometers, test candidates are asked to maintain a fixed speed, but must constantly adjust their effort to achieve a constant average speed. Reflecting this difficulty is obtaining steady state data, we utilise dynamical linear and nonlinear models.

\section{A. Linear model}

The linear model is of the form:

$$
\begin{aligned}
y(k) & =a_{0}+a_{1} y(k-1)+\ldots+a_{n a} y(k-n a) \\
& +b_{0} u(k-n k)+\ldots+b_{n b} u(k-n b-n k)
\end{aligned}
$$

Eqn. (1) articulates the familiar AutoRegressive with eXogenous input (ARX) model, with the exception that an affine term $a_{0}$ is included. This reflects the fact that a zero exercise intensity does not elicit a zero heart rate i.e the baseline resting heart rate is always superimposed. However, there are a number of factors, including an anticipatory increase in heart rate due to the onset of an intention to increase exercise intensity [13] and other transient phenomena, which result in a very small value for $a_{0}$, especially on the operational part of the steady state heart rate Vs exercise intensity curve. The structural parameters of Eqn. (1) which need to be determined are $n a$, the order of the output regressor, $n b$, the order of the input regressor and $n k$, the number of steps (sampling period increments) of pure delay between an increase in exercise and a corresponding reaction in heart rate. In this study, we employ Rissanen's minimum description length (MDL) [14] to determine $n a$ and $n b$ as 2 and 2 , respectively.

\section{B. Nonlinear model}

The nonlinear model has a form not dissimilar to that of Eqn. (1), with the exception that nonlinear terms in $y$ and $u$ 
are included, as:

$$
\begin{aligned}
y(k) & =a_{0}+a_{1,1} y(k-1)+\ldots+a_{1, n a} y(k-n a) \\
& +b_{1,0} u(k-n k)+\ldots+b_{1, n b} u(k-n b-n k) \\
& +a_{2,1} y(k-1)^{2}+\ldots+a_{2, n a} y(k-n a)^{2} \\
& +\ldots \\
& +a_{\text {oy }, 1} y(k-1)^{o y}+\ldots+a_{\text {oy }, n a} y(k-n a)^{\text {oy }} \\
& +b_{2,0} u(k-n k)^{2}+\ldots+b_{2, n b} u(k-n b-n k)^{2} \\
& +\ldots \\
& +b_{\text {ou }, 0} u(k-n k)^{\text {ou }}+\ldots+b_{\text {ou }, n b} u(k-n b-n k)^{\text {ou }}
\end{aligned}
$$

The additional structural parameters which must be determined for the NARX (nonlinear ARX) model in Eqn. (2) are $o y$, the max. polynomial order of the output terms and ou, the max. polynomial order of the input terms. We note that if oy $=1$, then the model in Eqn. (2) has a Hammerstein [15] structure, with the steady state response particularly simple to calculate. An orthogonal least squares algorithm for forward selection [16] was used to determine oy and ou as 2 and 2, respectively.

\section{Parameter identification}

A significant attraction of the nonlinear parameterisation in Eqn. (2) is the fact that the model, although nonlinear, is linear in the parameters. Therefore, we can employ simple identification techniques, such as least squares, to determine both linear and nonlinear model coefficients. For parameter identification, a variety of objective criteria can be used, including single-step or multi-step prediction criteria. While prediction error methods employing multi-step criteria [17] have been shown, in certain circumstances to have superior performance to single-step methods, the important factor in this study is the relative performance of linear and nonlinear models, rather than absolute model performance. Therefore, we employ a single-step criterion, permitting the use of simple batch least-squares for parameter identification, where the models represented by the equation:

$$
Y=\Phi \Theta+E
$$

are solved using the familiar formula:

$$
\hat{\Theta}=\left(\Phi^{T} \Phi\right)^{-1} \Phi^{T} Y+E
$$

where $E$ is the equation error and $\Phi$ and $\Theta$ are the regressor matrices and parameter vectors respectively, evaluated for Eqs. (1) and (2).

\section{Fuzzy model evaluation}

A number of options are available in relation to the fuzzy set shapes and how the unknown parameters, $p_{1}$ and $p_{2}$, are determined. Initially, we choose trapezoidal sets, in the absence of any specific knowledge to the contrary. A variety of line or concurrent search techniques can be employed for the determination of $p_{1}$ and $p_{2}$, depending on whether the overall performance surface to be searched is uni- or multi-modal. Initially, we employ a manual search, since there are just 2 parameters to determine and we wish to learn a little about the nature of the performance surface. To further simplify matters, we specify a constant interval between $p_{1}$ and $p_{2}$, resulting in just a single parameter (e.g. $\left(p_{1}+p_{2}\right) / 2$ to be determined. The $p_{2}-p_{1}$ interval was determined considering the rate of aerobic to anaerobic transition proposed in [18] and a value of $1.2 \mathrm{~km} / \mathrm{h}$ was employed.

\section{TEST PROTOCOLS}

In total, 9 candidates underwent a progressive loading test, with data logged initially on a per crank revolution, re-interpolated to a regularly sampled $0.5 \mathrm{~s}$ interval. The candidates were all male cyclists, aged 25 to 40 years old, with various levels of cycling ability. Each candidate was asked to follow an graded incremental test to volitional exhaustion, starting at $120 \mathrm{~W}$, with successive $30 \mathrm{~W}$ increments at 3 min intervals. Power variation was achieved via a cavitation adjustment using a fan window on a Wattbike (Wattbike Ltd., Nottingham, UK), while velocity was recorded and used as the measure of exercise intensity. A constant cadence, of 8384 RPM, was requested from test candidates.

\section{A. Data logged}

For each candidate, a wide variety of variables were recorded, including cadence, power, speed, heart-rate and a full respiratory gas analysis. A wide range of metabolic data was recorded using a Cosmed Quark $B^{2}$ respiratory analyser (Cosmed, Rome, Italy), providing a breath-by-breath measurement of volume and various gasses. These respiratory measurements were not used in the current analysis but may form the basis for future non-invasive AT tests. Fig.3 shows the evolution in speed and heart-rate for Candidate 2, by way of example. Lactate samples (from the ear) collected 2 min into each 3 min exercise element and analysed using a YSI1500 Sport Lactate Analyser (YSI, Ohio USA), which was pre-calibrated with a $5 \mathrm{mmol}$ lactate standard prior to each new candidate undertaking a test.

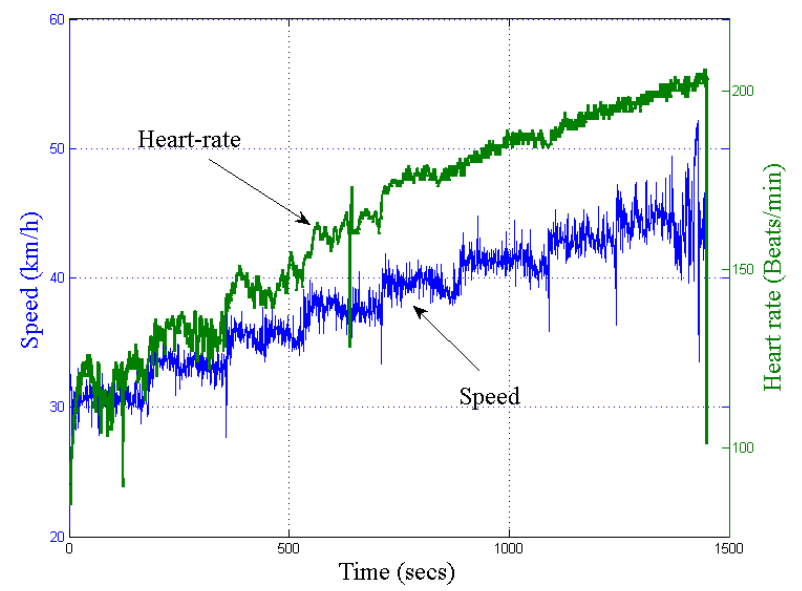

Fig. 3. Speed and heart rate evolution for Candidate 2

It is clear from Fig.3 that speed (or, indeed, power) is not nearly constant, with the variance increasing with 
increasing average velocity, due to the increased difficulty of maintaining a constant speed/power at high effort levels.

\section{B. Data preprocessing}

For much of the data, both heart-rate and speed signals contain some drop-outs, or outliers, and these must be removed prior to modelling. In general, such outliers occur as single point events, as evidenced from Fig.3, allowing a simple linear interpolation to be performed between immediately previous and following points to determine a reasonable value for the erroneous point.

\section{Lactate-based AT determination}

Fig.4 shows the lactate recordings, by way of example, for one of the test candidates (Candidate 3). The black dash-dot construction lines show the determination of AT as (Power,Lactate, HR) = (308(W),3.1(mmol/L),178(beats/min)). There are a few potential sources of error in such a determination:

- Portable lactate analysers, such as the YSI1500 have inherent, if small, measurement errors of the order of $\pm 0.5 \mathrm{mmol} / l$ [19],

- There are a relatively small number of lactate measurement points (typically 8-10) from which to derive asymptotes, and

- Since the lactate measurements are taken 2-min. into each effort incremental level and the power/speed is not constant (as evidenced from Fig.3), there may be inconsistencies in the lactate concentration measured at various nominal exercise intensities.

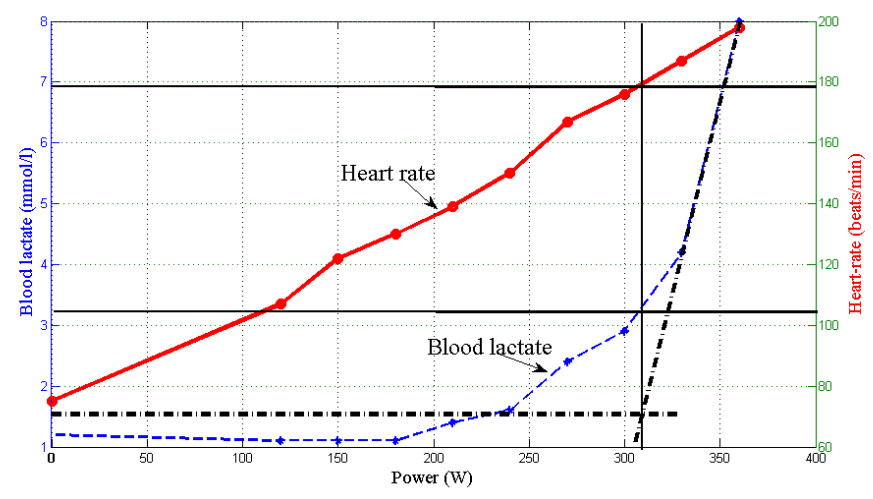

Fig. 4. Lactate test results for Candidate 3

In spite of these difficulties, AT determination using lactate measurements is still the gold standard.

\section{Results}

In this section, we show some individual modelling and fuzzy parameter determination results (Section IV-A), by way of example, while Section IV-B gives the summary results, which compare the AT results achieved using the fuzzy model to those measured using lactate analysis.

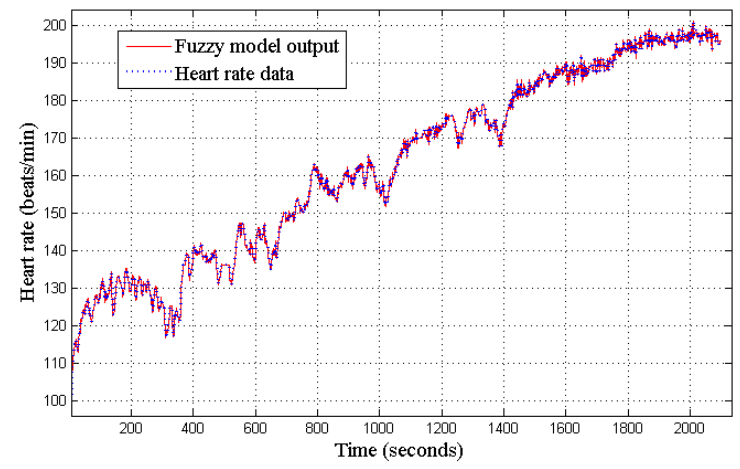

Fig. 5. Example fit (Candidate 4) of fuzzy model to recorded heart-rate data

\section{A. Sample individual results}

Fig.5 shows the fit of the fuzzy model to the measured heart rate data for Candidate 4. Clearly, the model provides a good fit over the full HR range, though it must be borne in mind that only 1-step prediction is performed by the model. However, Fig.5 validates the use of the 1-step criterion as a comparative measure for balancing the linear and nonlinear model components.

Fig.6 shows the variation in the fuzzy model MSE for Candidate 7 , as the centre point of the fuzzy parameters (i.e. $\left.\left(p_{1}+p_{2}\right) / 2\right)$ is varied, and is indexed according to speed. A value of AT, the transition point between the linear and nonlinear model, is indicated as $36 \mathrm{~km} / \mathrm{h}$. Note that the sensitivity of the MSE to variations in $\left.\left(p_{1}+p_{2}\right) / 2\right)$ reduces considerably in the region of the minimum point. In fact, the profile of the MSE variation with $\left.\left(p_{1}+p_{2}\right) / 2\right)$ was found to have a strong dependence on $\left(p_{2}-p_{1}\right)$.

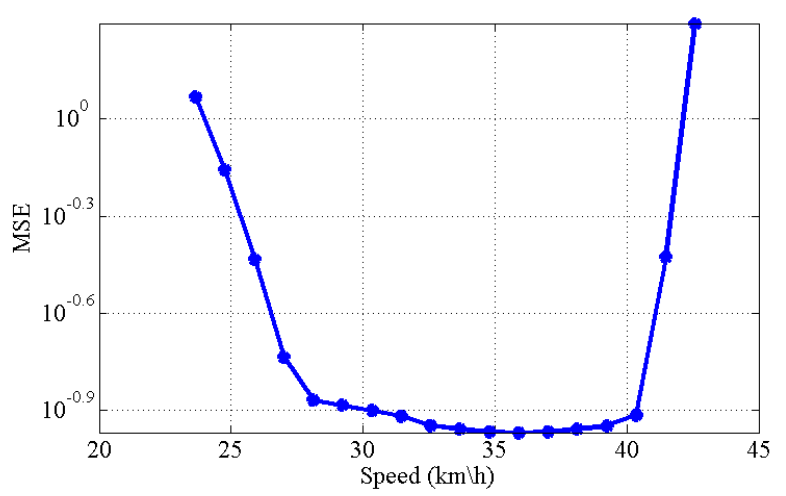

Fig. 6. Typical (Candidate 7) fuzzy model MSE profile variations with fuzzy crossover point

\section{B. Summary results}

Table I shows the comparative results achieved by the fuzzy parameter optimisation, compared to the reference measurement provided by lactate analysis. In general, the correlation is good and probably close to within the total experimental error of the lactate-derived AT measurements, considering the factors outlined in Section III-C. The mean 


\begin{tabular}{|c|c|c|c|c|c|c|c|c|c|}
\hline Candid. & 1 & 2 & 3 & 4 & 5 & 6 & 7 & 8 & 9 \\
\hline \hline Lactate & 184 & 170 & 163 & 174 & 155 & 156 & 160 & 157 & 173 \\
\hline Fuzzy & 180 & 160 & 159 & 170 & 155 & 151 & 160 & 170 & 167 \\
\hline
\end{tabular}

TABLE I

COMPARATIVE AT HEART-RATE VALUES FOR FUZZY- AND LACTATE-DETERMINED METHODS

difference between the AT values determined by lactate analysis and the fuzzy interpolation approach is 5.11 Beats/min and the correlation coefficient between the two measures is 0.77 over the 9 points. Broadly speaking, it can be reasonably concluded that the concept of using fuzzy interpolation between linear and nonlinear dynamical models to noninvasively determine AT has been proven.

\section{CONCLUSIONS}

The paper presents a non-invasive method to determine AT, based only on measurement of exercise intensity(speed/power) and heart rate. It appears to validate well against gold standard measurements made using invasive lactate analysis, with some explanation for the variance possibly attributable to the difficulty in obtaining an exact AT value from lactate measurements. While the results confirm the validity of the approach, there are a number of possible enhancements which could be considered, including independent optimisation of $p_{1}$ and $p_{2}$, employing a different measure of exercise intensity (e.g. power, instead of speed), use of a multi-step prediction error criterion, alternative fuzzy set shapes, etc.

Even if the AT determination using the fuzzy interpolation approach is not completely exact, the fact that it is noninvasive and requires, for the most part, readily available measurements, is a major advantage. In addition, the fact that the composite model is based on dynamical data places much less restriction on the type of experimental protocol required for AT determination. While a relatively rigid test protocol was employed for the data used in this study, necessitated by the requirement for steady-state lactate measurement, the fuzzy interpolation approach can be used with a much looser test protocol, extending its potential use to any situation where a protocol employing a generally monotonically increasing exercise intensity profile is used (since the forward and reverse dynamics of lactic acid production are different). This increases the accessibility of AT estimation for recreational athletes, for example for cyclists, runners, kayakers, rowers, etc where test conditions are controlled i.e. where no additional forces (e.g. wind, gradient, water current, etc) are present, without the explicit need for an ergometer.

\section{REFERENCES}

[1] E. Fox, Sports Physiology, 2nd ed. Saunders, 1984.

[2] J. Wilmore and D. Costill, Physiology of Sport and Exercise, 3rd ed. Human Kinetics, 2005.

[3] S. Lowe and G. ÓLaighin, "The age of the virtual trainer," Procedia Engineering, vol. 34, pp. 242-247, 2012.

[4] V. L. Billat, P. Sirvent, G. Py, J.-P. Koralsztein, and J. Mercier, "The concept of maximal lactate steady state," Sports Medicine, vol. 33, no. 6, pp. 407-426, 2003.

[5] K. M. d. Souza, T. Grossl, B. Junior, R. José, R. D. d. Lucas, V. P. Costa, and L. G. A. Guglielmo, "Maximal lactate steady state estimated by different methods of anaerobic threshold," Revista Brasileira de Cineantropometria \& Desempenho Humano, vol. 14, no. 3, pp. 264-275, 2012.

[6] K. Tanaka, Y. Matsuura, S. Kumagai, A. Matsuzaka, K. Hirakoba, and K. Asano, "Relationships of anaerobic threshold and onset of blood lactate accumulation with endurance performance," European Journal of Applied Physiology and Occupational Physiology, vol. 52, no. 1, pp. 51-56, 1983.

[7] F. Conconi, M. Ferrari, P. G. Ziglio, P. Droghetti, and L. Codeca, "Determination of the anaerobic threshold by a noninvasive field test in runners," Journal of Applied Physiology, vol. 52, no. 4, pp. 869873, 1982.

[8] F. Conconi, G. Grazzi, I. Casoni, C. Guglielmini, C. Borsetto, E. Ballarin, G. Mazzoni, M. Patracchini, and F. Manfredini, "The Conconi test: methodology after 12 years of application," International Journal of Sports Medicine, vol. 17, no. 07, pp. 509-519, 1996.

[9] A. Jones and J. Doust, "Lack of reliability in conconi's heart rate deflection point," International Journal of Sports Medicine, vol. 16, no. 08 , pp. 541-544, 1995

[10] J. Bourgois and J. Vrijens, "The Conconi test: a controversial concept for the determination of the anaerobic threshold in young rowers," International Journal of Sports Medicine, vol. 19, no. 08, pp. 553$559,1998$.

[11] J. Ringwood, "Anaerobic threshold measurement using dynamic neural network models," Computers in Biology and Medicine, vol. 29, no. 4, pp. 259-271, 1999.

[12] T. Takagi and M. Sugeno, "Fuzzy identification of systems and its applications to modeling and control," IEEE Transactions on Systems, Man and Cybernetics, no. 1, pp. 116-132, 1985.

[13] A. Guyton and J. Hall, Textbook of Medical Physiology, 11th ed. Elsevier Saunders, 2006.

[14] J. Rissanen, Minimum-Description-Length Principle. Wiley, 1985.

[15] E. Eskinat, S. H. Johnson, and W. L. Luyben, "Use of hammerstein models in identification of nonlinear systems," AIChE Journal, vol. 37, no. 2, pp. 255-268, 1991.

[16] S. Chen, S. A. Billings, and W. Luo, "Orthogonal least squares methods and their application to non-linear system identification," International Journal of control, vol. 50, no. 5, pp. 1873-1896, 1989.

[17] L. Ljung, "Prediction error estimation methods," Circuits, Systems and Signal Processing, vol. 21, no. 1, pp. 11-21, 2002.

[18] A. Lucía, O. Sánchez, A. Carvajal, and J. L. Chicharro, "Analysis of the aerobic-anaerobic transition in elite cyclists during incremental exercise with the use of electromyography." British Journal of Sports Medicine, vol. 33, no. 3, pp. 178-185, 1999.

[19] J. Aduen, W. K. Bernstein, T. Khastgir, J. Miller, R. Kerzner, A. Bhatiani, J. Lustgarten, A. S. Bassin, L. Davison, and B. Chernow, "The use and clinical importance of a substrate-specific electrode for rapid determination of blood lactate concentrations," JAMA, vol. 272, no. 21 , pp. 1678-1685, 1994. 